\title{
Functional Connectivity Alterations of the Temporal Lobe and Hippocampus in Semantic Dementia and Alzheimer's Disease
}

\author{
Simon Schwab ${ }^{\mathrm{a}, \mathrm{d}}$, Soroosh Afyouni ${ }^{\mathrm{a}}$, Yan Chen ${ }^{\mathrm{b}}$, Zaizhu Han ${ }^{\mathrm{c}}$, Qihao Guo ${ }^{\mathrm{c}}$, Thomas Dierks ${ }^{\mathrm{d}}$, \\ Lars-Olof Wahlund ${ }^{\mathrm{e}}$ and Matthias Grieder ${ }^{\mathrm{d}, *}$ \\ ${ }^{a}$ Big Data Institute, Li Ka Shing Centre for Health Information and Discovery, Nuffield Department of Population \\ Health, University of Oxford, Oxford, United Kingdom \\ ${ }^{\mathrm{b}}$ State Key Laboratory of Cognitive Neuroscience and Learning, Beijing Normal University, Beijing, China \\ ${ }^{\mathrm{c} D e p a r t m e n t ~ o f ~ N e u r o l o g y, ~ H u a s h a n ~ H o s p i t a l, ~ F u d a n ~ U n i v e r s i t y, ~ S h a n g h a i, ~ C h i n a ~}$ \\ ${ }^{\mathrm{d}}$ Translational Research Center, University Hospital of Psychiatry and Psychotherapy, University of Bern, Bern, \\ Switzerland \\ ${ }^{\mathrm{e}}$ Department of Neurobiology, Care Sciences and Society, Karolinska Institutet, Division of Clinical Geriatrics, \\ Stockholm, Sweden
}

Handling Associate Editor: Claudio Babiloni

Accepted 27 May 2020

\begin{abstract}
.
Background: Semantic memory impairments in semantic dementia are attributed to atrophy and functional disruption of the anterior temporal lobes. In contrast, the posterior medial temporal neurodegeneration found in Alzheimer's disease is associated with episodic memory disturbance. The two dementia subtypes share hippocampal deterioration, despite a relatively spared episodic memory in semantic dementia.

Objective: To unravel mutual and divergent functional alterations in Alzheimer's disease and semantic dementia, we assessed functional connectivity between temporal lobe regions in Alzheimer's disease $(n=16)$, semantic dementia $(n=23)$, and healthy controls $(n=17)$.

Methods: In an exploratory study, we used a functional parcellation of the temporal cortex to extract time series from 66 regions for correlation analysis.

Results: Apart from differing connections between Alzheimer's disease and semantic dementia that yielded reduced functional connectivity, we identified a common pathway between the right anterior temporal lobe and the right orbitofrontal cortex in both dementia subtypes. This disconnectivity might be related to social knowledge deficits as part of semantic memory decline. However, such interpretations are preferably made in a holistic context of disease-specific semantic impairments and functional connectivity changes.
\end{abstract}

\footnotetext{
${ }^{*}$ Correspondence to: Matthias Grieder, $\mathrm{PhD}$, Translational Research Center, University Hospital of Psychiatry and Psychotherapy, Bolligenstrasse 111, 3000 Bern 60, Switzerland. Tel.: +41 319328351; Fax: +41 319309961; E-mail: matthias.grieder@upd.unibe.ch.
} 
Conclusion: Despite a major limitation owed to unbalanced databases between study groups, this study provides a preliminary picture of the brain's functional disconnectivity in Alzheimer's disease and semantic dementia. Future studies are needed to replicate findings of a common pathway with consistent diagnostic criteria and neuropsychological evaluation, balanced designs, and matched data MRI acquisition procedures.

Keywords: Alzheimer's disease, functional connectivity, semantic dementia, temporal lobe

\section{INTRODUCTION}

Everybody occasionally experiences difficulties in integrating past events into an accurate context-a condition classified as an episodic memory disturbance. Intact episodic memory [1] requires the processing of information about chronology, place, and the protagonists who were involved in an event. The capability to store and retrieve autobiographical memory is, however, not sufficient for an intact episodic memory. Humans also strongly rely on a fully functioning semantic memory. Concretely, semantic memory reflects our general knowledge about concepts such as objects, people, and words. Thus, only a sound interplay of these two memory systems, episodic and semantic memory, allows a cognitively healthy state of an individual.

Previously two initially contradicting models of the neurophysiological organization of semantic memory have been harmonized as what can be characterized as a "cortically distributed plus semantic hub' theory [2, 3]. The term "distributed" refers to the idea that regions which process semantic concepts receive multimodal input from corresponding brain regions (e.g., visual attributes from visual brain regions, tactile attributes from the sensorimotor cortex, etc.). Subsequently, these multimodal inputs from distributed cortical areas converge to so-called unitary semantic concepts in the semantic hub $[4,5]$. The semantic hub was found to be localized bilaterally in the anterior temporal lobe, a region which is atrophied and hypometabolized in patients with the semantic variant of primary progressive aphasia, also known as the temporal variant of frontotemporal dementia (FTD) or semantic dementia (SD) [5-7]. In $\mathrm{SD}$, the onset of gray matter atrophy occurs in the anterior temporal lobes, frequently with an asymmetry toward the more affected left hemisphere. With progression of the disease, the temporal pole and medial as well as lateral temporal areas are degenerated [8]. However, the patients seem to exhibit an almost intact episodic memory, when tested nonverbally, while their semantic memory is severely deteriorated $[9,10]$.
In contrast to $\mathrm{SD}$, patients with Alzheimer's disease (AD) show predominantly episodic memory impairments, and semantic memory deficits can only be observed to a minor degree [11-13]. AD has been described as a disconnection syndrome, that is, connections of functionally or structurally linked brain regions that are part of a network become increasingly disrupted [14-16]. This degenerative mechanism has been associated with the cognitive deficits of patients with $\mathrm{AD}$ [17-19]. A common finding in $\mathrm{AD}$ is that gray matter atrophy onset can be localized in the hippocampal, posterior cingulate, and lateral parietal brain regions, as well as in the amygdala [20, 21]. The hippocampus forms a core region for episodic memory encoding. However, it has also been associated with semantic memory functions [22]. In fact, Burianova and colleagues [22] postulated that the hippocampus is part of a common declarative memory network, suggesting that the hippocampus has a key role in both semantic as well as episodic and autobiographical memory.

The properties of functional systems, as for example Burianova and colleagues' proposed declarative memory network, are assessed by the use of a resting-state functional connectivity (FC) analysis. The human resting-state is characterized by spatially discriminate brain regions that co-activate and deactivate at a low temporal frequency, commonly known as resting-state networks [23, 24]. These functional systems, or resting-state networks, are assessed using blood-oxygen level dependent resting-state fMRI. It has become very popular to study FC alterations in various mental and neurological disorders including $\mathrm{AD}$, demonstrating a relationship between disease and abnormalities in resting-state networks [25-27].

FC changes (i.e., decreases and increases of connectivity strengths) in AD have been found predominantly in the hippocampus and the default mode network [28-31]. With the progression of the disease, structural and functional connectivity distortions affect several networks, particularly those involving the parahippocampal gyrus [17, 32]. In $\mathrm{SD}, \mathrm{FC}$ appears to be deteriorated in regions either 
affected by or proximate to the core of atrophy, located in regions such as the temporal pole, anterior middle temporal gyrus, inferior temporal gyrus, and insula [6,33-35]. Furthermore, reduced FC of the anterior temporal lobe with various cortical regions was also found in SD [2].

Considering these findings as well as the distinct pathology of $\mathrm{AD}$ and $\mathrm{SD}$, it is likely that the neuronal loss of hippocampal cells that results in gray matter atrophy affects the functional networks in a way that generates episodic memory deficits. Temporal pole atrophy alone might not be necessary (but sufficient) to lead to semantic impairment. Following these findings, La Joie et al. [36] identified the hippocampus as the 'main crossroad' between brain networks that are disrupted in AD and SD. Despite the growing body of research, the common and divergent changes of FC among regions of the temporal lobes in $\mathrm{AD}$ and $\mathrm{SD}$ are not fully understood. A caveat when interpreting the existing literature is the use of anatomical/structural parcellation instead of a functional parcellation to study FC. Functional parcellations have the advantage that the resulting functional regions of interest (ROIs) are homogeneous, i.e., the voxels have similar time courses. On the other hand, parcellations based on brain structure can merge the time series across functionally different areas, which can be problematic [37].

This proof-of-concept study aimed at disentangling FC alterations in the temporal lobe in $\mathrm{AD}$ and SD using a refined division of temporal subregions: sixty-six functional ROIs of the temporal lobes from a functional atlas [38]. In contrast to numerous previous studies, we accounted for structural changes (i.e., gray matter density) in order to extract FC time series data from preserved gray matter tissue which can still be functional $[39,40]$. In other words, results from the FC analysis reflect the functional reorganization of the temporal lobes affected by atrophy.

A common issue with studies involving patients with SD is the small sample size due to the low prevalence and relatively difficult diagnosis. In order to overcome this to some extent, we pooled two data sets from two different recording sites (see Method section for details). Orban et al. [41] showed the advantage of multisite fMRI-data in multivariate fMRI analysis. Their approach appears to be generalizable; however, in our study, we were not able to accomplish an evenly matched number of patients or controls at each MRI scanner site, which is a prerequisite for a correct experimental design. In particular, the circumstance that the majority of SD patients was scanned at the Shanghai site and all AD patients and healthy controls (HC) were scanned at the Stockholm site, increases the likelihood of false positive contrasts between the groups due to instrumental artifacts. Other inherent limitations will be addressed in the discussion section (e.g., site-specific diagnostic criteria, neuropsychological testing, and fMRI acquisition procedures).

Despite the exploratory analysis approach to test all possible connections, based on previous findings described above, the following hypotheses were tested: in $\mathrm{AD}$, we expected $\mathrm{FC}$ alterations in the hippocampus, parahippocampal ROIs, and possibly posterior temporal ROIs. In SD, altered FC was anticipated in the hippocampus, the fusiform gyrus, and the temporal pole.

\section{METHODS}

\section{Participants}

We analyzed resting-state fMRI data from a total of 62 participants from three groups: semantic dementia (SD), Alzheimer's disease (AD), and a healthy elderly control group (HC). We examined all the functional MRI data and excluded six datasets due to insufficient data quality (see data quality control). The final sample consisted of 56 participants: Twenty-three patients with SD, with a mean age ( \pm standard deviation) of $61.5 \pm 7.4,16$ patients with $\mathrm{AD}$, mean age of $68.4 \pm 8.5$, and 17 individuals in the $\mathrm{HC}$ group, mean age $67.9 \pm 3.3$; see Table 1 for demographics and clinical variables. Patients with SD from the Stockholm site $(n=7)$ were recruited throughout Sweden and diagnosed using the criteria of Neary et al. [42], while patients with SD from Shanghai were recruited from Huashan Hospital in Shanghai $(n=19)$, according to the criteria of Gorno-Tempini et al. [43]. The main diagnostic criteria of both guidelines share clinical observation features such as impaired word naming and comprehension, spared repetition, and surface dyslexia and dysgraphia. Differences in these two diagnostic criteria, as for instance the introduction of brain imaging as a supportive diagnostic feature in GornoTempini et al. [43], were not relevant, because also the Swedish patients underwent MRI to assess anterior temporal lobe atrophy. Patients with AD were recruited at the Memory Clinic of the Geriatric Department at Karolinska University Hospital in Huddinge, Sweden $(n=19)$. Their diagnosis was performed by expert clinicians and was in accordance 
Table 1

Descriptives and clinical scores. Kruskal-Wallis tests were run to assess group differences of age, education, MMSE, BNT, lexical decision, AF, and VF. Comparisons between AD and SD of the CDS and GDS scores were performed using the non-parametric Kolmogorov-Smirnov-Test

\begin{tabular}{|c|c|c|c|c|c|}
\hline & $\frac{\mathrm{HC}(n=17)}{\text { Mean (std. dev. })}$ & $\begin{array}{l}\text { Normative data }^{\dagger} \\
\text { Mean (std. dev.) }\end{array}$ & $\begin{array}{c}\mathrm{AD}(n=16) \\
\text { Mean (std. dev.) }\end{array}$ & $\frac{\mathrm{SD}(n=23)}{\text { Mean (std. dev. })}$ & $p$ \\
\hline Age, y & $67.9(3.3)$ & & $68.4(8.5)$ & $61.5(7.4)$ & 0.004 \\
\hline Gender (F:M) & $12: 5$ & & $7: 9$ & $10: 13$ & - \\
\hline Education, y & $13.9(3.1)$ & & $13.1(3.0)$ & $12.4(1.5)^{3}$ & 0.61 \\
\hline CDS & - & & $1.0(1.0)$ & $1.8(2.2)^{3}$ & 0.60 \\
\hline GDS & - & & $2.9(0.8)$ & $3.8(0.4)^{3}$ & 0.031 \\
\hline MMSE (max 30) & $28.8(0.8)$ & & $24.5(4.8)$ & $20.8(5.2)^{4}$ & $<0.0001$ \\
\hline BNT $(\max 60)$ & $54.4(3.7)$ & $54.0(4.5)$ & $45.6(6.5)$ & $8.2(5.7)^{3}$ & $<0.0001$ \\
\hline Oral picture-naming $(\max 140)$ & - & & - & $39.2(27.6)^{5}$ & - \\
\hline Word-triple association $(\max 70)$ & - & & - & $51.2(10.1)^{5}$ & - \\
\hline Number calculation task $(\max 7)$ & - & & - & $6.36(1.1)^{5}$ & - \\
\hline Lexical decision $(\max 352)$ & $346.0(3.7)^{1}$ & & $333.2(23.5)^{2}$ & $325.3(23.0)^{6}$ & 0.002 \\
\hline $\mathrm{AF}$, animals/min & $23.8(5.9)$ & $18.2(3.8)$ & $14.1(4.2)$ & $5.6(4.3)^{3}$ & $<0.0001$ \\
\hline $\mathrm{VF}$, verbs/min & $21.9(5.8)$ & $18.2(5.6)$ & $11.9(5.0)$ & $7.0(2.8)^{3}$ & $<0.0001$ \\
\hline
\end{tabular}

${ }^{\dagger}$ Normative data are reference values for comparison of the control group (HC) with respect to BNT with $\mathrm{N}=32$ [81]; AF with $\mathrm{N}=94$ [82]; VF with $N=67$ [83]. ${ }^{1} n=16,{ }^{2} n=12,{ }^{3} n=5,{ }^{4} n=19,{ }^{5} n=14,{ }^{6} n=4$. CDS, Cornell Depression Scale; GDS, Global Deterioration Scale; MMSE, Mini-Mental State Examination; BNT, Boston Naming Test; AF, animal fluency; VF, verb fluency.

with the ICD-10 criteria [44]. The patients with AD included in this study underwent a standard clinical procedure which consisted of examinations such as structural neuroimaging, lumbar puncture, blood analyses, and neuropsychological assessment (these assessments were part of the clinical routine and only used for diagnosis). Further inclusion criteria for patients from the Stockholm site was a Global Deterioration Scale lower than 6 (i.e., moderate dementia or milder) and the Cornell Depression Scale below 8 . Healthy elderly controls were recruited by advertisement $(n=22)$ in the Stockholm area. Presence of medical or psychiatric disorders (other than dementia), intake of drugs affecting the nervous system, or magnetic implants, led to an exclusion from the study. Variables available for all participants included in the study were age, gender, and Mini-Mental State Examination (MMSE).

All study participants provided informed consent prior to the data acquisition. The Shanghai study was approved by the Institutional Review Board of the State Key Laboratory of Cognitive Neuroscience and Learning, Beijing Normal University [33]. The Stockholm study was approved by the Regional Ethics Committee of Stockholm, Sweden.

\section{MRI data}

MR images were acquired at two sites: The Karolinska Institute in Stockholm, Sweden, and the Huashan Hospital in Shanghai, China.

\section{Stockholm site}

MR images were acquired with a 3T Siemens Magnetom Trio scanner (Siemens AG, Erlangen, Germany). Structural images were 3D T1-weighted magnetization-prepared rapid gradient echo (MPRAGE) images using the following parameters: $\mathrm{TR}=1900 \mathrm{~ms}, \mathrm{TE}=2.57 \mathrm{~ms}$, flip angle $=9^{\circ}$, matrix size $=256 \times 256$, field of view $=230 \times 230 \mathrm{~mm}^{2}$, slice number $=176$ slices, slice thickness $=1 \mathrm{~mm}$, and voxel size $=0.90 \times 0.90 \times 1 \mathrm{~mm}^{3}$. The structural images were previously used for voxel-based morphometry and published with a different purpose and sample configuration [45, 46]. Functional images were acquired with a 32-channel head coil, using an interleaved EPI sequence (400 volumes; 26 slices; voxel, $3 \times 3 \times 4 \mathrm{~mm}^{3}$; gap thickness, $0.2 \mathrm{~mm}$; matrix size, $80 \times 80$; FOV, $240 \times 240 \mathrm{~mm}^{2}$; TR, $1600 \mathrm{~ms}$; TE, $35 \mathrm{~ms}$ ).

\section{Shanghai site}

Images were acquired with a 3T Siemens Magnetom Verio. Structural images were 3D T1-weighted magnetization-prepared rapid gradient echo (MPRAGE) images using the following parameters: $\mathrm{TR}=2300 \mathrm{~ms}, \mathrm{TE}=2.98 \mathrm{~ms}$, flip angle $=9^{\circ}$, matrix size $=240 \times 256$, field of view $=240 \times 256 \mathrm{~mm}^{2}$, slice number $=192$ slices, slice thickness $=1 \mathrm{~mm}$, and voxel size $=1 \times 1 \times 1 \mathrm{~mm} 3$. Functional images were acquired with a 32-channel head coil, using an interleaved EPI sequence (200 volumes; 33 slices; voxel, $4 \times 4 \times 4 \mathrm{~mm}^{3}$; gap thickness, $0 \mathrm{~mm}$; matrix size, $64 \times 64$; FOV, $256 \times 256 \mathrm{~mm}^{2}$; TR, $2000 \mathrm{~ms}$; 
TE, $35 \mathrm{~ms}$, flip angle $90^{\circ}$ ). The data were previously published with a different sample configuration (SD only sample) in a combined structural and functional study using a hippocampus seed region [47], as well as in a structural voxel-based morphometry (VBM) study [33].

\section{Preprocessing of functional MRI scans}

We performed pre-processing using SPM12 (http://www.fil.ion.ucl.ac.uk/spm). We initially set all images' origin to the anterior commissure, and then performed slice-time correction, realignment, coregistration, normalization to MNI space $\left(2 \times 2 \times 2 \mathrm{~mm}^{3}\right)$, and smoothing (full width half maximum [FWHM]; $8 \mathrm{~mm}$ ). Time series data were high-pass filtered $(1 / 128 \mathrm{~Hz})$ and we regressed out 14 nuisance parameters (6 movement parameters and their first derivative, white matter, and cerebrospinal fluid).

We carefully assessed data quality and inspected the spatio-temporal quality of each scan by comparing the slow and fast components of the data using DSE (Dvar, Svar\&Evar) decomposition [48]. The DSE technique decomposes the dataset into three main components: fast, which is the squared mean difference; slow, which is the squared mean averages, and Evar, which refers to the sum of squares of the two ends of the time series. Subjects with remarkably high divergence (>75\%-tile) between Dvar and Svar components were removed, as suggested in Afyouni \& Nichols [48]. Therefore, we removed one SD and three $\mathrm{HC}$ datasets from the analysis. We further excluded two AD subjects, as more than $20 \%$ of their DVARS data-point were found to be corrupted. The remaining subjects were scrubbed as suggested by Power et al. [49]. Altogether, we excluded six datasets $(9.7 \%)$ due to poor data quality. We re-run the diagnostics on the final sample and found no difference between groups regarding the DSE diagnostics (oneway ANOVA, all $p>0.05$ ).

\section{Functional connectivity analysis}

We investigated FC between each of the 66 temporal ROIs in three participant groups $(\mathrm{AD}, \mathrm{SD}$, and $\mathrm{HC}$ ). We focused our analysis on the temporal lobes with the following rationale: first, brain regions identified as the origin of atrophy are located in the temporal lobe. Second, a 'crossroad' of FC network disruption in $\mathrm{AD}$ and $\mathrm{SD}$ was found in the hippocampus. Third, functional hubs for episodic and semantic memory can be found in the temporal lobe (as outlined above). Fourth, the strongest FC of temporal regions is located within the temporal lobes and concurs with functional networks crucial for language processing, the core clinical feature of SD [50]. The functional parcellation we used is based on restingstate fMRI data which was clustered into spatially coherent regions of homogeneous FC and was evaluated in terms of the generalizability of group level results to the individual [38]. From the 200 ROIs, we used a subset of 66 temporal ROIs that covered at least $5 \%$ or more of one of the following temporal structures from WFU Pickatlas 3.0.4 [51]: the superior temporal cortex, the middle temporal cortex, the inferior temporal cortex, the temporal pole, the hippocampus, the parahippocampal cortex, the lingual gyrus, the amygdala, the insular cortex, and the fusiform gyrus; these 66 ROIs are shown in Supplementary Figure 1. Analyzing merely 66 temporal ROIs leads to 2,145 pair wise correlations, which necessitates a strong adjustment for multiple comparisons to control for false positives. Using an even higher number of ROIs, for example comparing 200 ROIs in the whole brain, would require an even stronger correction (correcting for almost 20,000 comparisons). Such corrections would result in a sensitivity too low to detect even substantial FC changes.

We extracted the mean time series from the gray matter (probability $>0.70$ ) of these ROIs to assure that time series were not contaminated with cerebrospinal fluid signals from atrophied areas, resulting in 66 time series per subject. We also assured that time series were not affected by signal dropouts due to dephasing. To address motion and physiological confounds which are global in nature, we applied global signal regression to the time series [52-54]. We created a pair-wise correlation matrix and transformed the correlation coefficient to Z-scores by Fisher's transformation. We conducted a one-way ANOVA for each ROI pair (2,145 tests) to test the null hypothesis of no difference between the three groups. We performed an additional sensitivity analysis with age, mean gray matter density in the temporal cortex, MMSE, and study site as additional covariates. Covariates can be problematic if these differ between groups [55], therefore we report these sensitivity analyses in the Supplementary Material. From the 2,145 total connections, we found 321 (sensitivity analysis: 324$)$ significant edges that showed a group effect (uncorrected, $p<0.05$ ), and after correcting the $p$-values for multiple comparisons, seven edges 
showed a significant group effect (FDR corrected, $p<0.05$ ).

\section{Voxel-based morphometry analysis}

We additionally performed a VBM analysis to quantify gray matter loss in the patients from the anatomical T1 images. VBM is a voxel-wise comparison of the local amount of gray matter volume between two groups [56]. We performed the following processing steps: spatial registration to MNI space (voxel size: $1.5 \times 1.5 \times 1.5 \mathrm{~mm}^{3}$ ) and tissue segmentation, bias correction of the intensities, smoothing of the GM images with $8 \mathrm{~mm}$ FWHM, and modulation by scaling with the total volume so that the resulting amount of gray matter in the modulated images remained the same as in the native images. In other words, this step removed the introduced bias from the registration of different brain sizes to MNI space. The Stockholm sample was registered using the European brain template, the Shanghai sample with the East Asian brain template and normalized to MNI space. We used the "Computational Anatomy Toolbox" CAT12 [57] and SPM12 [58] for the VBM analysis. Statistical inference was performed with the "Statistical Non Parametric Mapping" software SnPM13 using non-parametric permutation/randomization two-sample $t$-tests with a voxel-wise family-wise error correction (FWE) of 0.05 . We performed two $t$-tests and compared the HC group versus the SD group, and the $\mathrm{HC}$ group versus the $\mathrm{AD}$ group. Unlike in the analyses of the functional data where we excluded six datasets, the structural T1 scan from all the subjects were used in this analysis, the group sizes were HC with $n=20$, AD with $n=18$, SD with $n=24$.

\section{RESULTS}

We first describe the clinical presentation of the patients included in this study (see Table 1 for details). The SD group performed poorer in MMSE than the AD group (Kruskal-Wallis over all groups: $H=29.5$, $\mathrm{df}=2, p<0.0001$; Kolmogorov-Smirnov group-wise post-hoc tests: HC-AD $Z=1.86, p=0.002$, HC-SD $Z=2.52, p<0.001, \mathrm{AD}-\mathrm{SD} Z=1.44, p=0.033$ ). Furthermore, the SD group showed significantly lower scores in the Boston Naming Test (BNT) than the AD group (Kruskal-Wallis over all groups: $H=23.3$, $\mathrm{df}=2, p<0.0001$; Kolmogorov-Smirnov group-wise post-hoc tests: HC-AD $Z=1.85, p=0.002$, HC$\mathrm{SD} Z=1.97, p=0.001$, AD-SD $Z=1.95, p=0.001)$.
Within the SD group, we observed that the impaired performance in picture naming (BNT, Stockholm site; oral picture-naming, Shanghai site) were more pronounced than lexical decision (Stockholm site) and word-triple association (Shanghai site), see Table 1. The group differences between SD and AD in MMSE and BNT are common findings given that the BNT is a semantic task and the MMSE relies on language comprehension, as both semantics and language are typically more affected in SD than AD. Finally, our AD group also showed semantic deficits as compared to the healthy control group (based on BNT, animal fluency, and verbal fluency). These behavioral scores mirror the severe semantic memory deficits in patients with SD. Moreover, the normal calculation ability in the majority of our SD group supported the diagnostic features of SD. In contrast, patients with AD showed a comparably mild semantic memory deficit, which is in accordance with the expectations. MMSE was the only available neuropsychological test score for all participants from both sites, whereas the remaining tests were sitespecific and therefore not comparable.

Next, we report the gray matter density found in the patient groups, see Fig. 1. In the SD patients (Fig. 1A), we found two clusters of atrophy. The first was located in the left anterior medial temporal cortex, with a peak effect in the left temporal fusiform cortex (peak $t$-score $=14.0, p_{\mathrm{FDR}}=0.0021, \mathrm{df}=42$; location at $x=-34, y=-3, z=-36$; cluster area $80.7 \mathrm{~cm}^{3}$ ). The second cluster was located in the temporal fusiform cortex of the right hemisphere (peak $t$-score $=10.6$, $p_{\mathrm{FDR}}=0.0021, \mathrm{df}=42 ;$ location at $x=34, y=-3$, $z=-34$; cluster area $40.1 \mathrm{~cm}^{3}$ ). In the AD patients, we found two clusters with lower GM volume compared to controls in the left amygdala (peak $t$-score $=8.72$, $p_{\text {FDR }}=0.006, \mathrm{df}=36$; location at $x=-26, y=-10$, $z=-12$; cluster area $7.23 \mathrm{~cm}^{3}$ ) and the right amygdala (peak $t$-score $=7.49, p_{\text {FDR }}=0.006, \mathrm{df}=36$; location at $x=22, y=-3, z=-15$; cluster area $7.47 \mathrm{~cm}^{3}$ ), see Fig. 1B. A commonly expected hippocampal atrophy was yielded only with a more liberal threshold (Supplementary Figure 2).

To achieve the main goal of this study, we analyzed the functional connectivity of 56 participants using 66 functional ROIs of the temporal cortex and related sub cortical areas (see complete correlational matrix in Supplementary Figure 3). Seven connections (FC between ROI pairs) demonstrated a significant difference between the three groups after correcting for multiple comparisons (FDR corrected, $p<0.05$ ). A detailed characterization and test statistics of these 


\section{A Semantic dementia GM atrophy}
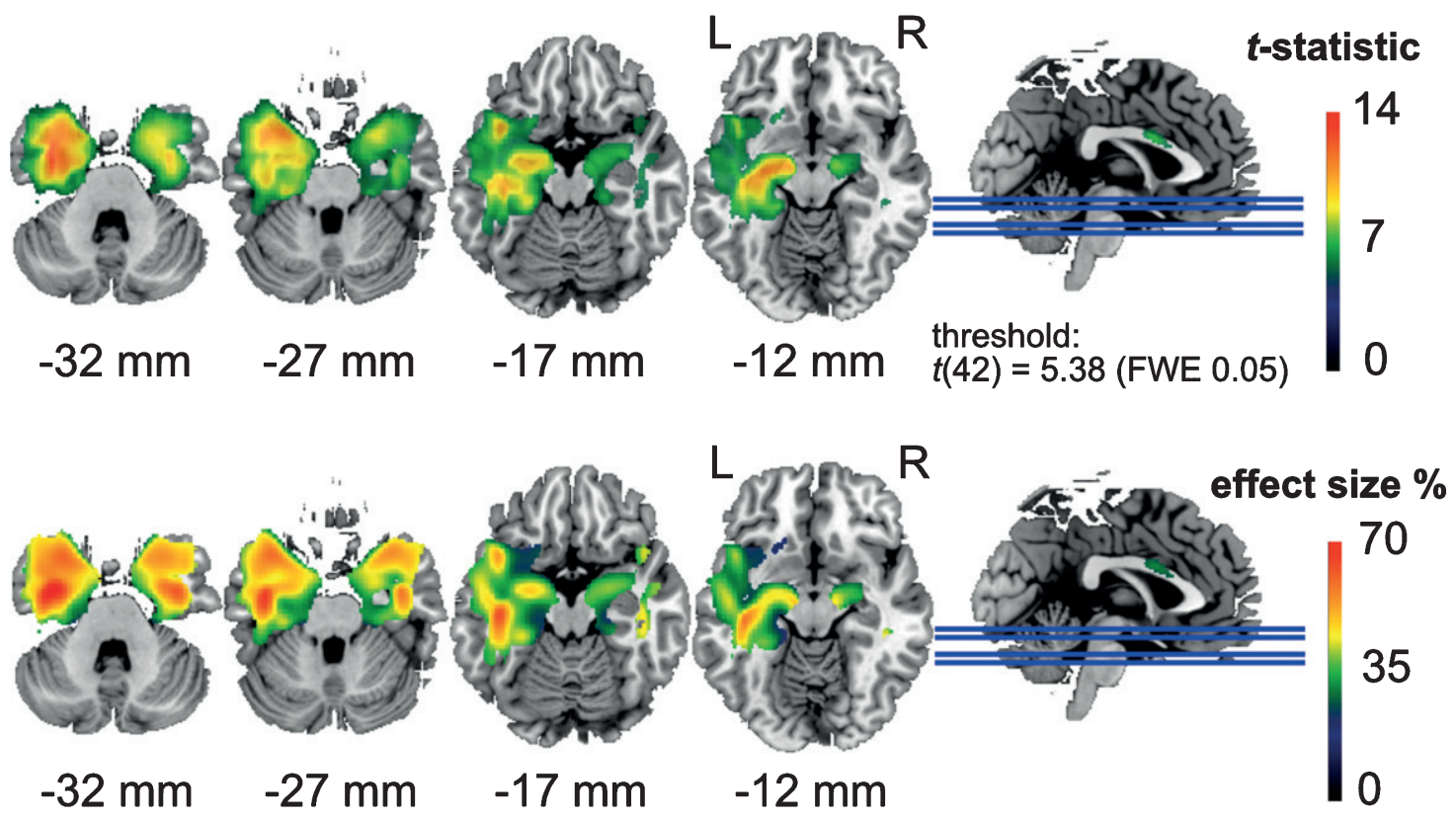

\section{B Alzheimer's disease GM atrophy}
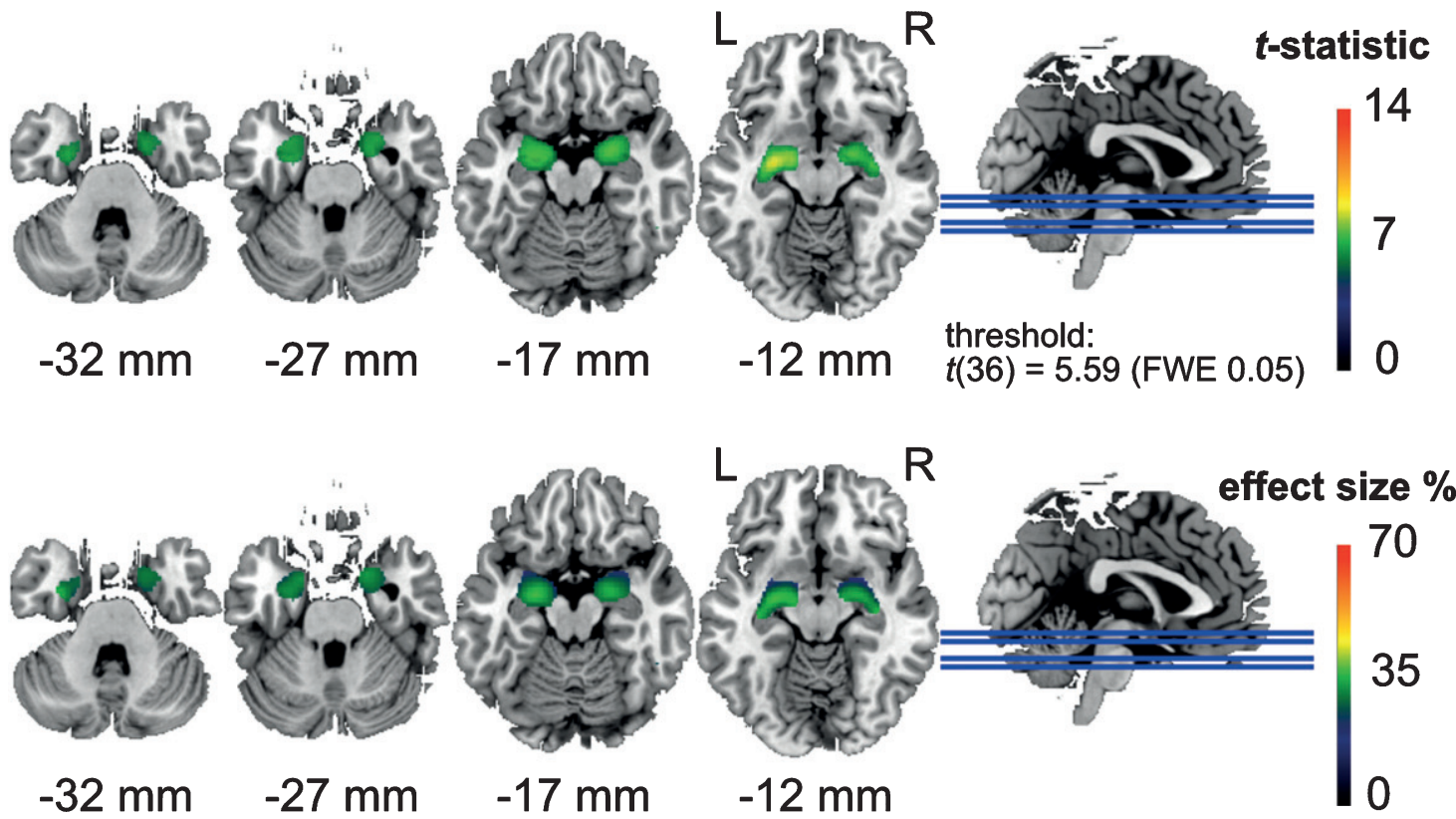

Fig. 1. Areas with significantly lower (voxel-level) gray matter (GM) density (top) and effect size in terms of percentage GM reduction (bottom) in (A) the semantic dementia (SD) patients $(n=24)$ and (B) Alzheimer's disease (AD) patients $(n=18)$ compared to the healthy elderly control group $(n=20)$. SD patients showed reduced GM density in widespread areas of the left anterior temporal cortex including the temporal pole, while the AD patients showed reduced GM density in the amygdala. SD patients showed more severe GM loss with up to $70 \%$ reduction, and $\mathrm{AD}$ patients with up to $40 \%$ reduction in some areas. 
Table 2

Seven functional connections that demonstrated significant group differences

\begin{tabular}{|c|c|c|c|c|c|c|}
\hline Edge no. & ROI no. & ROI no. & Region & Region & $F$ & FDR $\operatorname{adj} . p$ \\
\hline 1 & 129 & 11 & $\begin{array}{l}\text { Left anterior superior temporal } \\
\text { gyrus/middle temporal } \\
\text { gyrus/insular cortex }\end{array}$ & $\begin{array}{l}\text { Left posterior middle temporal } \\
\text { gyrus/superior temporal gyrus }\end{array}$ & 10.86 & 0.034 \\
\hline 2 & 85 & 24 & $\begin{array}{l}\text { Right lateral inferior occipital } \\
\text { cortex/lateral superior occipital } \\
\text { cortex }\end{array}$ & $\begin{array}{l}\text { Left posterior superior temporal } \\
\text { gyrus/central opercular } \\
\text { cortex/parietal opercular } \\
\text { cortex/planum temporale }\end{array}$ & 11.52 & 0.030 \\
\hline 3 & 198 & 32 & $\begin{array}{l}\text { Right fusiform } \\
\text { cortex/parahippocampal gyrus }\end{array}$ & Right inferior temporal pole & 12.64 & 0.026 \\
\hline 4 & 70 & 37 & $\begin{array}{l}\text { Left lingual gyrus/intracalcarine } \\
\text { cortex/precuneus cortex }\end{array}$ & Left posterior hippocampus/thalamus & 13.18 & 0.026 \\
\hline 5 & 89 & 37 & $\begin{array}{l}\text { Right lingual gyrus/intracalcarine } \\
\text { cortex }\end{array}$ & Left posterior hippocampus/thalamus & 10.95 & 0.034 \\
\hline 6 & 153 & 71 & $\begin{array}{l}\text { Right anterior middle temporal } \\
\text { gyrus/superior temporal gyrus }\end{array}$ & Right orbitofrontal cortex & 12.42 & 0.026 \\
\hline 7 & 112 & 72 & $\begin{array}{l}\text { Left orbitofrontal cortex/insular } \\
\text { cortex }\end{array}$ & $\begin{array}{l}\text { Left anterior inferior temporal } \\
\text { gyrus/middle temporal gyrus }\end{array}$ & 12.06 & 0.026 \\
\hline
\end{tabular}
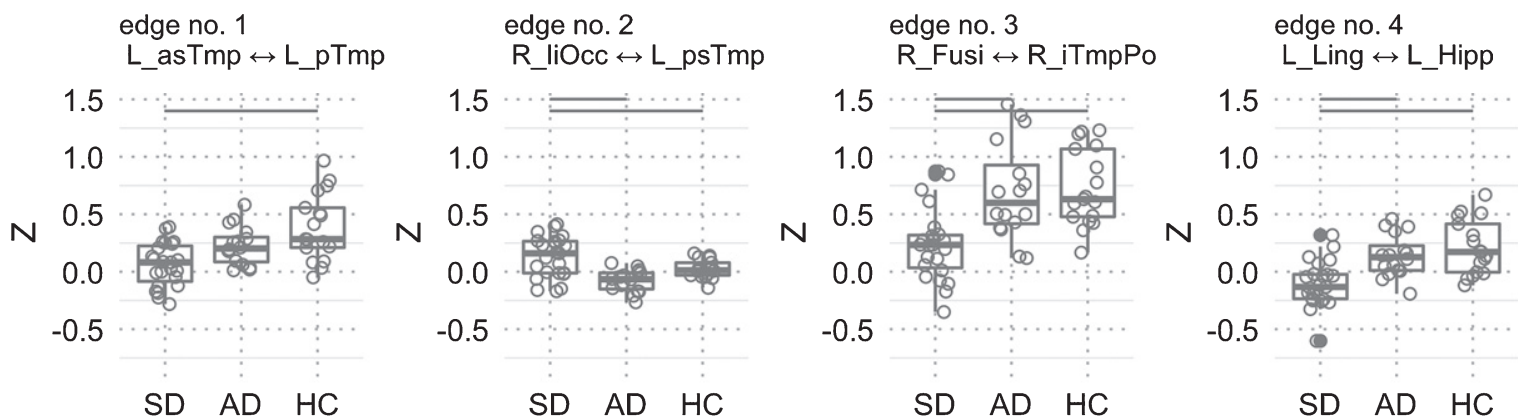

SD AD HC

edge no. 5

R_Ling $\leftrightarrow$ L_Hipp

\section{edge no. 6}

R_mTmp $\leftrightarrow$ R_FrtOrb

\section{edge no. 7}
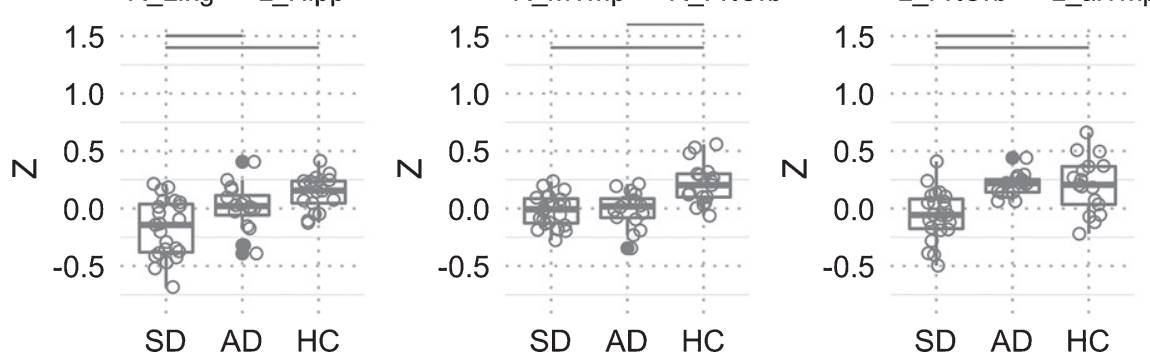

Fig. 2. Z-scores of seven connections (edges 1-7) with significant group differences. Post-hoc tests between the three groups were performed, and significant group differences are denoted with gray horizontal lines (see Table 2 for a detailed description of the ROIs). Ring-shaped circles represent single subject data points. Filled circles represent outliers.

Table 3

Post-hoc Tukey HSD $p$-values for the three single comparisons (rows) and for each of the seven ROI-pairs that had a significant group effect (columns). Significant values reflect that the group effect was driven by a specific group level contrast

\begin{tabular}{lccccccc}
\hline Edge No. & 1 & 2 & 3 & 4 & 5 & 6 & 7 \\
\hline AD versus HC & 0.098 & 0.082 & 0.98 & 0.54 & 0.22 & 0.0005 & 1.00 \\
SD versus HC & $<0.0001$ & 0.044 & 0.0002 & $<0.0001$ & $<0.0001$ & $<0.0001$ & 0.0004 \\
SD versus AD & 0.064 & $<0.0001$ & 0.0004 & 0.002 & 0.024 & 0.97 & 0.0004 \\
\hline
\end{tabular}

seven connections are shown in Table 2; the Z-values for the significant connections are depicted in Fig. 2. We performed post-hoc tests (Tukey HSD) for sin- gle comparisons of the three groups to investigate the particular group contrasts that drove the significant group effect (Table 3). We found that most differ- 
ences were related to the SD patients with significant changes in all of the seven connections. SD patients showed lower FC in 6 out of 7 connections compared to $\mathrm{HC}$, and higher FC in one connection (edge no. 2) compared to HC. This higher FC in the SD patients was also significantly higher compared to the $\mathrm{AD}$ patients. The AD patients had a lower FC compared to $\mathrm{HC}$ patients in only 1 out of the 7 connections (edge 6). Comparing the two patient groups SD, versus $\mathrm{AD}$, we found that $\mathrm{SD}$ had a significant lower FC in 4 connections (edges 3, 4, 5, 7).

We visualized the connectivity structure and connection strengths, see Fig. 3. The SD patients generally had a much lower connectivity compared to the other two groups. An exception was the stronger contralateral connection between the right lateral inferior occipital cortex and the left posterior superior temporal gyrus (edge no. 2). The AD patients showed a lower FC compared to HC between the right middle temporal gyrus and the right frontal orbital cortex (edge no. 6). A common finding in all the three groups was that the FC between the right fusiform cortex and the right inferior temporal pole was the strongest (no. 3).

The sensitivity analysis with age, mean gray matter density in the temporal cortex, MMSE, and study site as covariates produced statistically significant differences in the same seven edges as reported above; however, the assumption of the ANCOVA, the independence between the patient group and the covariates was not met. For results of the sensitivity analysis with covariates, see Supplementary Tables 1-4.

\section{DISCUSSION}

In this study, we compared functional connectivity between $\mathrm{SD}, \mathrm{AD}$, and $\mathrm{HC}$ using a functional parcellation of 66 ROIs of the temporal cortex and hippocampus to investigate intra-temporal connections and connections with contralateral temporal regions. The overall picture that emerges is that between the majority of the significant ROIs, SD demonstrated the most striking decrease in FC. In the $\mathrm{AD}$ group, most differences compared to the $\mathrm{HC}$ group did not reach significance. We believe that the often described disconnections found in AD were not detected in our study due to the mild progression of the disease in our $\mathrm{AD}$ group. One reason could be that the remaining gray matter volume in brain regions typically affected by neuronal degeneration was suffi- cient to maintain an intact FC to remote areas. In other words, the damage found in mild stages might affect the intra-regional processing in local neuronal populations, whereas the inter-regional (i.e., network) FC would be affected during more advanced AD progression [59]. Future studies will require larger sample sizes to demonstrate smaller changes in FC seen even with mild state impairments.

The most intriguing finding of our study for the SD group was the decreased FC between the left posterior hippocampus and left/ right lingual gyri (edges 4 and 5). These disruptions are characteristic for the neurophysiological basis of the SD patients' typical symptomatology involving an impaired semantic memory. For instance, Sormaz et al. [60] recently showed a correlation of FC between left the hippocampus and the lingual gyrus with topographic memory, and a correlation of semantic memory performance with FC to the intracalcarine cortex, a finding consistent with our results.

Functional connectivity between the left anterior superior/middle temporal gyrus/insula and the left posterior middle/superior temporal gyrus was decreased in SD compared to HC (edge no. 1). It is important to note that this is the single connection that showed an FC difference between SD and $\mathrm{HC}$ exclusively (i.e., a finding specific for the SD$\mathrm{HC}$ group single-comparison while neither AD-HC nor AD-SD were significant). These regions are commonly associated with cross-modal integration (as is the hypothesized semantic hub) of auditory and language processing, as well as the processing of the emotionally relevant context. Hence, this finding might reflect the severe semantic deficits in SD (see Table 1) that are manifested by the loss of conceptual knowledge [7].

The single connection that showed increased FC in SD compared to the other groups (edge no. 2) was between the left posterior superior temporal gyrus/parietal opercular cortex/planum temporale and the right lateral inferior/superior occipital cortex. The temporal brain areas that constitute this connection are important for early context integration of acoustically presented words [61], lexico-semantic retrieval [62], and are part of a supramodal semantic network [63]. The occipital ROI of this connection subserves visual integration. Thus, an increased FC between these regions might reflect a functional reorganization that is characterized by supporting language comprehension using more sensory inputs. Moreover, this result indicated a reduced hemispheric functional specialization and perhaps an attempt to 


\section{Semantic dementia (SD)}
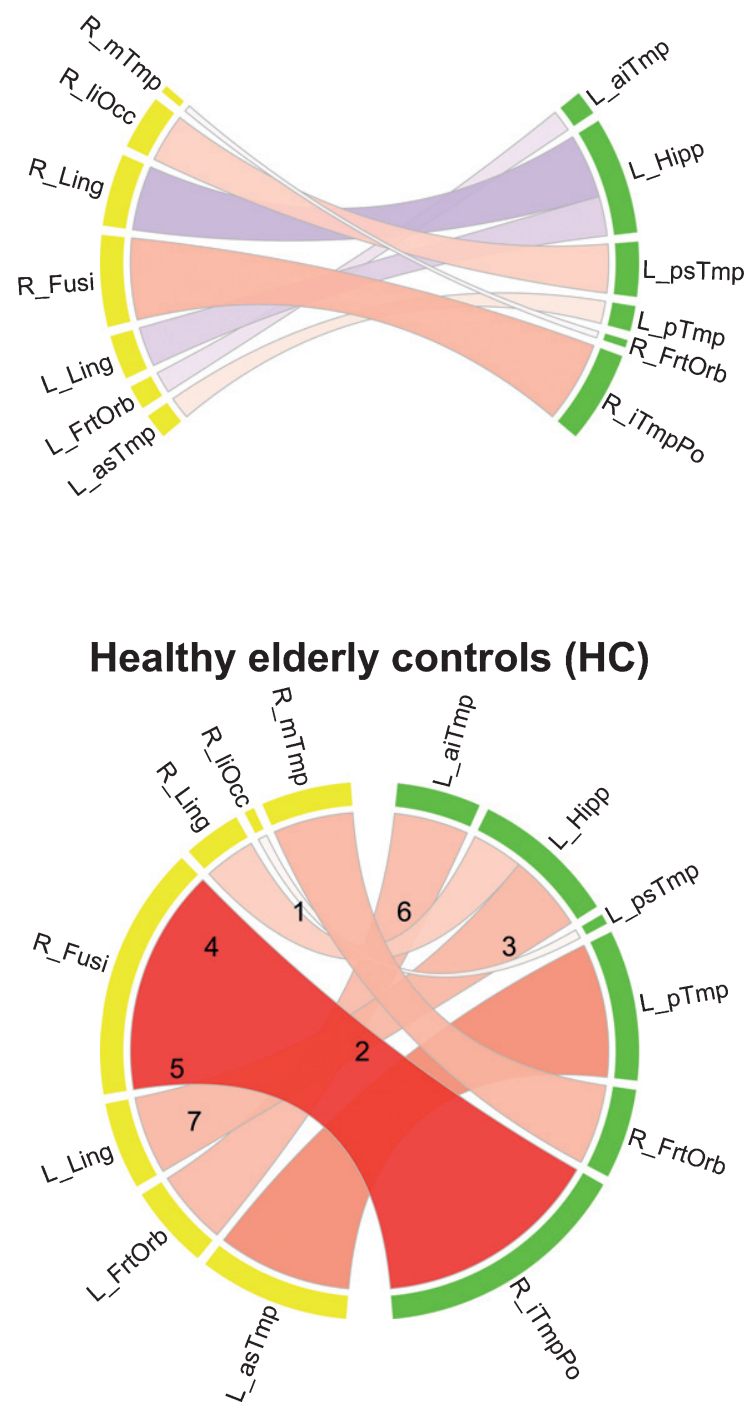

\section{Alzheimer's disease (AD)}

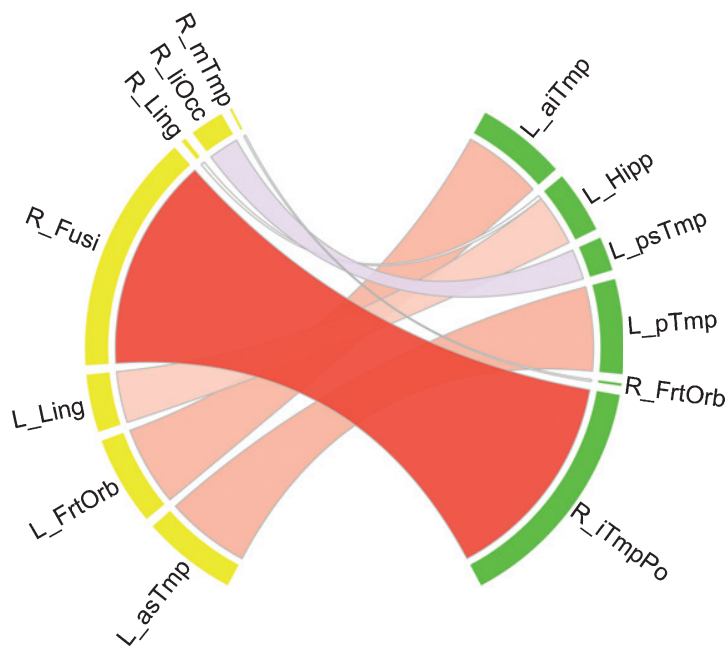

Fig. 3. Functional connectivity (FC) strengths of the three groups. Color shades and thickness of the links are proportional to FC strengths; shades of red reflect positive, shades of blue negative strengths. Numbers in HC group indicate edge numbers (see Table 2 for a detailed description of the ROIs). ROIs in the left hemisphere are labeled yellow, ROIs in the right hemisphere labeled green.

pool resources that are spared by the pathological developments in SD.

In comparison with $\mathrm{AD}$ and $\mathrm{HC}, \mathrm{SD}$ patients showed a lower FC between the functional ROI encompassing the right fusiform/parahippocampal gyri and the right inferior temporal pole. Disruption of this connection (no. 3) can be viewed as SD-typical, as the functional profile of the involved regions conforms to SD symptomatology. In particular, the right temporal pole is crucial for non-verbal (e.g., visual) object recognition, which is a hallmark impairment in SD associated with the loss of semantic knowledge [64, 65]. The right fusiform gyrus on the other hand is associated with working memory for faces, face perception, and non-verbal associative semantic knowledge [66-68], and the right parahippocampal gyrus is associated with working memory for object location as well as a function as an episodic buffer $[69,70]$. In line with this, the patients with SD in the present study showed severe object recognition 
deficits assessed with BNT and oral picture-naming (Table 1), even though no behavioral data about face perception or non-verbal semantic knowledge was available.

Similar to connection no. 3, FC was reduced in $\mathrm{SD}$ compared with $\mathrm{AD}$ and $\mathrm{HC}$ between the ROI comprising left lingual/intracalcarine/precuneus cortex and the ROI including left posterior hippocampus/thalamus (connection no. 4). This finding is in line with Seeley et al. [14], who reported the medial temporal lobe as part of an SD-vulnerable network. Thus, in addition we showed a possible contribution of the primary visual (intracalcarine cortex), visual memory (lingual gyrus) and self-awareness (precuneus, i.e., default mode network) regions to that semantic network. It might appear surprising that the $\mathrm{FC}$ of the AD group was not significantly reduced in this connection, despite the commonly known medial temporal lobe atrophy and the pivotal role of the hippocampus in episodic memory encoding [36, 71]. However, functional and anatomical changes do not necessarily overlap, and for instance, stable FC of the left hippocampus in early AD (except with right lateral prefrontal cortex) has been reported previously [29].

Lower $\mathrm{FC}$ in $\mathrm{SD}$ than in $\mathrm{AD}$ (and $\mathrm{HC}$ ) was also found between the right lingual/intracalcarine cortex and the left posterior hippocampus/thalamus (connection no. 5). Therefore, connections between bilateral lingual gyri and the left hippocampus were detected in our HC sample (for illustration, see Fig. 3, connections no. 4 and 5), whereas either of them were damaged in SD, but not in AD. This supports the recent indication of a hippocampal contribution to the semantic memory network [36]. Because episodic memory is relatively spared in SD, the connections between the left posterior hippocampus and the bilateral lingual gyri might contribute to the semantic memory network. On the other hand, we did not find an expected decrease of FC in connection no. 4 in $\mathrm{AD}$, although the precuneus and hippocampus contribute to episodic memory, which is typically impaired in AD. However, we have to bear in mind that our analysis was restricted to temporal lobe FC and thus did not cover the entire episodic memory network, including brain regions located in frontal and parietal lobes. In addition, no episodic memory data were available for the entire sample of our study. Future studies should investigate additional ROIs from the aforementioned areas using larger sample sizes to tackle the increased number of connections and multiple testing corrections that are associated with larger networks.
The only FC reduction common to both SD and $\mathrm{AD}$ compared with $\mathrm{HC}$ was found in connection no. 6 . The functional role of the involved regions suggests an association with a frequently observed clinical presentation of $\mathrm{AD}$ and $\mathrm{SD}$ characterized by apathy and agitation, associated with the right orbitofrontal cortex [72, 73], and impairments in social behavior related to the right anterior temporal lobe [74]. According to Olson et al. [75], social knowledge is part of semantic memory and involves memory about people including biographical information. Nonetheless, caution is advised with comparing social or semantic deficits between $\mathrm{AD}$ and SD; both symptoms have different onsets or severities within disease stages, as well as different characteristics. Furthermore, we did not have data on social behavior or apathy/agitation of our patients. Regardless, we added a common pathway to the crossroad described by La Joie et al. [36]. They suggested that the hippocampus is a converging hub of an (AD-affected) episodic and a (SD-affected) semantic network. Accordingly, our data indicated that besides a shared damaged hub in $\mathrm{AD}$ and $\mathrm{SD}$, the functional connection between the right anterior middle/superior temporal gyri and the right orbitofrontal cortex might be a second candidate for the neuropathology shared in both clinical populations.

The final significant connection (no. 7) of the present study was found between the left orbitofrontal cortex and the left anterior inferior and middle temporal gyri. The literature suggests a functional role of this connection in deficient socioemotional abilities that are found predominantly in the behavioral variant of FTD [76], and in higher level object representation, involving language and auditory processing. Unlike in connection no. 6, the AD group did not show an impaired FC of the orbitofrontal regions with the ipsilateral temporal cortex. Thus, one might speculate about a bilateral breakdown of orbitofrontal to temporal connections in SD, which might be related to the severity of the semantic deficit.

This study entailed a number of study design limitations that need to be taken into account while interpreting the results. Even though the overall sample size is large, the sample sizes of the three subgroups are considered small (16-23 individuals). Larger studies need to be conducted, however, this is especially challenging for SD given its low prevalence. Therefore, we pooled two SD samples from two different sites with different scanners. However, most individuals of the SD group and none of the $\mathrm{AD}$ and $\mathrm{HC}$ groups were from the Shanghai site, 
which is a violation of acknowledged study design standards and a potential confound. Moreover, the diagnostic criteria of the two sites for SD were not identical. The data from different MRI sites may have different noise levels such as thermal noise, physiological noise, and motion [52, 77]. These artifacts are often global in nature, and global signal regression (GSR) can successfully remove these and standardize the data between sites and across individuals. GSR can introduce negative correlations; however, GSR can also improve the specificity of positive correlations [78]. Importantly, in this study, we do not interpret absolute negative correlations and solely compare relative differences in correlations between groups. We conducted a sensitivity analysis with the study site as covariate which yielded the same results. However, assumptions of independence between the covariates and the patient groups were not met. The present limitation of the unbalanced study design cannot entirely be removed by an analysis of covariance. Thus, future studies should measure different patient populations across different scanner sites and ideally achieve balanced groups across sites. Harmonization techniques [79] can further improve data quality. However, the application of harmonization methods in unbalanced groups is questionable as these not only eliminate scanner effects, but also the effects of interest [80]. Moreover, interpretation of group differences between $\mathrm{AD}$ and SD should take into account that the two dementia groups were not matched for disease stage (i.e., SD showed more severe deficits than AD). The sensitivity analysis with GM density as covariate was in line with our results. Likewise, more symptom specific behavioral scores (other than MMSE) could have aided an in-depth interpretation of altered FC edges in the patient groups. Lastly, our analysis did not cover all brain regions potentially relevant for AD and SD. However, the choice to limit the scope to the temporal lobe has three reasons: first, the distinct temporal lobe atrophy is crucial for AD and SD differentiation. Second, the temporal lobe is pivotal in both semantic and episodic memory functions. Third, the definition of ROIs within the temporal lobe, even though using an arbitrary selection threshold of $5 \%$ (or more) of overlap of the ROIs with any temporal structure, may be altogether less arbitrary and biased compared to subjectively selecting ROIs based on expectations and literature.

To summarize the main findings of our study, the cohort of patients with SD yielded a number of distinct ipsilateral and contralateral connections of the temporal lobe that showed a significant reduc- tion in FC. These connections included the regions on which our predictions were based on (i.e., hippocampus, fusiform gyrus, and temporal pole). Two functional connections were intriguing due to their distinctiveness from the other groups: the first was the connectivity breakdown between left posterior hippocampus and bilateral lingual gyri, likely reflecting the neuronal underpinning of semantic memory loss. Second, a bilateral disruption of connectivity between temporal and frontal lobes was found. This aligns well with the pathophysiology within the FTD spectrum and especially with SD.

FC in AD was relatively intact compared to SD, which contradicted our hypothesis. The only connection with significantly reduced FC encompassed the right orbitofrontal cortex and the right anterior temporal lobe (no. 6), which we identified as an AD/SD-common pathway. Additionally, Fig. 2 illustrates that our AD group had a lower FC than the HC in connections no. 1,2 , and to a smaller extent no. 5 which all missed significance. These FC signatures in the $\mathrm{AD}$ group could be attributed to their mild stage of symptom progression (MMSE of 24.5), and potentially an early marker of the disease, but larger and longitudinal studies are needed.

Following the "cortically distributed plus semantic hub" theory, several connections were found to be significantly altered in the present study, which affected the anterior temporal lobe - semantic hub regions (no. 1, 3, 6, and 7). Moreover, their counterparts were partly localized in the modality-specific regions described by Patterson et al. [5], but also in orbitofrontal regions. This agreement of our results with the arguments in Patterson et al. [5] supported the "distributed plus hub" theory, because we found altered FC in connections between the hub and the modality-specific regions. Taken together, this study presents an alternative concept to investigate the understanding of distinct pathophysiological changes in $\mathrm{AD}$ and SD that are related to disruptions of functional networks in the temporal lobe. The unique aspect of our study was the definition of ROIs based on functional brain segregation rather than anatomy for FC analysis. Due to the comparably strict statistical approach and the predefined choice of ROIs, our study provided a fine-grained overview of FC aberration related to temporal lobe function in $\mathrm{AD}$ and SD. However, comparability was limited owing to different study sites using partially different diagnostic criteria and data acquisition procedures. We emphasize here that this was an exploratory study with the motivation of gathering MRI data of a rare 
condition (SD) from two study sites to increase statistical power. The downside of this approach was that the retrospective characteristic caused unbalanced recording of the study groups across the two MRI scanning sites. This required the conduction of several control analyses to mitigate the occurrence of false contrasts between the groups. Thus, our findings ideally motivate future studies for replication with harmonized MRI acquisition parameters and balanced subject numbers between study sites, concurring with an optimal research practice.

\section{DATA AVAILABILITY}

Raw imaging data can be requested from the corresponding author. Aggregated data and analysis scripts to generate all results and figures are available at OSF (https://osf.io/t4jnv/).

\section{ACKNOWLEDGMENTS}

This work was supported by the Swedish Alzheimer fonden, the Swiss Synapsis Foundation, and the University of Bern, the Beijing Natural Science Foundation (7182088), and the NSFC (31872785). Simon Schwab acknowledges funding from the Swiss National Science Foundation (SNSF, No. 162066 and 171598).

Authors' disclosures available online (https:// www.j-alz.com/manuscript-disclosures/19-1113r2).

\section{SUPPLEMENTARY MATERIAL}

The supplementary material is available in the electronic version of this article: https://dx.doi.org/ 10.3233/JAD-191113.

\section{REFERENCES}

[1] Tulving E (1983) Elements of Episodic Memory, Oxford University Press, Oxford.

[2] Guo CC, Gorno-Tempini ML, Gesierich B, Henry M, Trujillo A, Shany-Ur T, Jovicich J, Robinson SD, Kramer JH, Rankin KP, Miller BL, Seeley WW (2013) Anterior temporal lobe degeneration produces widespread network-driven dysfunction. Brain 136, 2979-2991.

[3] Mummery CJ, Patterson K, Price CJ, Ashburner J, Frackowiak RS, Hodges JR (2000) A voxel-based morphometry study of semantic dementia: Relationship between temporal lobe atrophy and semantic memory. Ann Neurol 47, 36-45.

[4] McClelland JL, Rogers TT (2003) The parallel distributed processing approach to semantic cognition. Nat Rev Neurosci 4, 310-322.

[5] Patterson K, Nestor PJ, Rogers TT (2007) Where do you know what you know? The representation of semantic knowledge in the human brain. Nat Rev Neurosci 8, 976-987.

[6] Hodges JR, Patterson K (2007) Semantic dementia: A unique clinicopathological syndrome. Lancet Neurol 6, 1004-1014.

[7] Landin-Romero R, Tan R, Hodges JR, Kumfor F (2016) An update on semantic dementia: Genetics, imaging, and pathology. Alzheimers Res Ther 8, 52.

[8] Collins JA, Montal V, Hochberg D, Quimby M, Mandelli ML, Makris N, Seeley WW, Gorno-Tempini ML, Dickerson BC (2017) Focal temporal pole atrophy and network degeneration in semantic variant primary progressive aphasia. Brain 140, 457-471.

[9] Bozeat S, Lambon Ralph MA, Patterson K, Garrard P, Hodges JR (2000) Non-verbal semantic impairment in semantic dementia. Neuropsychologia 38, 1207-1215.

[10] Irish M, Bunk S, Tu S, Kamminga J, Hodges JR, Hornberger M, Piguet O (2016) Preservation of episodic memory in semantic dementia: The importance of regions beyond the medial temporal lobes. Neuropsychologia 81, 50-60.

[11] Blackwell AD, Sahakian BJ, Vesey R, Semple JM, Robbins TW, Hodges JR (2004) Detecting dementia: Novel neuropsychological markers of preclinical Alzheimer's disease. Dement Geriatr Cogn Disord 17, 42-48.

[12] MascaliD, DinuzzoM, Serra L, Mangia S, Maraviglia B, Bozzali M, Giove F (2018) disruption of semantic network in mild Alzheimer's disease revealed by resting-state fMRI. Neuroscience 371, 38-48.

[13] Vogel A, Gade A, Stokholm J, Waldemar G (2005) Semantic memory impairment in the earliest phases of Alzheimer's disease. Dement Geriatr Cogn Disord 19, 75-81.

[14] Seeley WW, Crawford RK, Zhou J, Miller BL, Greicius MD (2009) Neurodegenerative diseases target large-scale human brain networks. Neuron 62, 42-52.

[15] Villain N, Fouquet M, Baron J-C, Mézenge F, Landeau B, de La Sayette V, Viader F, Eustache F, Desgranges B, Chételat G (2010) Sequential relationships between grey matter and white matter atrophy and brain metabolic abnormalities in early Alzheimer's disease. Brain 133, 3301-3314.

[16] Wang J, Zuo X, Dai Z, Xia M, Zhao Z, Zhao X, Jia J, Han Y, He Y (2013) Disrupted functional brain connectome in individuals at risk for Alzheimer's disease. Biol Psychiatry 73, 472-481.

[17] Liu J, Zhang X, Yu C, Duan Y, Zhuo J, Cui Y, Liu B, Li K, Jiang T, Liu Y (2016) Impaired parahippocampus connectivity in mild cognitive impairment and Alzheimer's disease. J Alzheimers Dis 49, 1051-1064.

[18] Reijmer YD, Leemans A, Caeyenberghs K, Heringa SM, Koek HL, Biessels GJ, Utrecht Vascular Cognitive Impairment Study Group (2013) Disruption of cerebral networks and cognitive impairment in Alzheimer disease. Neurology 80, 1370-1377.

[19] Tijms BM, Yeung HM, Sikkes SAM, Möller C, Smits LL, Stam CJ, Scheltens P, van der Flier WM, Barkhof F (2014) Single-subject gray matter graph properties and their relationship with cognitive impairment in early and late-onset Alzheimer's disease. Brain Connect 4, 337-346.

[20] Buckner RL, Snyder AZ, Shannon BJ, LaRossa G, Sachs R, Fotenos AF, Sheline YI, Klunk WE, Mathis CA, Morris JC, Mintun MA (2005) Molecular, structural, and functional characterization of Alzheimer's disease: Evidence for a relationship between default activity, amyloid, and memory. $J$ Neurosci 25, 7709-7717.

[21] Poulin SP, Dautoff R, Morris JC, Barrett LF, Dickerson BC, Alzheimer's Disease Neuroimaging Initiative (2011) 
Amygdala atrophy is prominent in early Alzheimer's disease and relates to symptom severity. Psychiatry Res 194, 7-13.

[22] Burianova H, McIntosh AR, Grady CL (2010) A common functional brain network for autobiographical, episodic, and semantic memory retrieval. Neuroimage 49, 865-874.

[23] Biswal B, Yetkin FZ, Haughton VM, Hyde JS (1995) Functional connectivity in the motor cortex of resting human brain using echo-planar MRI. Magn Reson Med 34, 537-541.

[24] Raichle ME, MacLeod AM, Snyder AZ, Powers WJ, Gusnard DA, Shulman GL (2001) A default mode of brain function. Proc Natl Acad Sci U S A 98, 676-682.

[25] Broyd SJ, Demanuele C, Debener S, Helps SK, James CJ, Sonuga-Barke EJS (2009) Default-mode brain dysfunction in mental disorders: A systematic review. Neurosci Biobehav Rev 33, 279-296.

[26] Zhang D, Raichle ME (2010) Disease and the brain's dark energy. Nat Rev Neurol 6, 15-28.

[27] Greicius MD, Srivastava G, Reiss AL, Menon V (2004) Default-mode network activity distinguishes Alzheimer's disease from healthy aging: Evidence from functional MRI. Proc Natl Acad Sci U S A 101, 4637-4642.

[28] Allen G, Barnard H, McColl R, Hester AL, Fields JA, Weiner MF, Ringe WK, Lipton AM, Brooker M, McDonald E, Rubin CD, Cullum CM (2007) Reduced hippocampal functional connectivity in Alzheimer disease. Arch Neurol 64, 1482-1487.

[29] Wang L, Zang Y, He Y, Liang M, Zhang X, Tian L, Wu T, Jiang T, Li K (2006) Changes in hippocampal connectivity in the early stages of Alzheimer's disease: Evidence from resting state fMRI. Neuroimage 31, 496-504.

[30] Tahmasian M, Pasquini L, Scherr M, Meng C, Förster S, MulejBratec S, Shi K, Yakushev I, Schwaiger M, Grimmer T, Diehl-Schmid J, Riedl V, Sorg C, Drzezga A (2015) The lower hippocampus global connectivity, the higher its local metabolism in Alzheimer disease. Neurology 84, 1956-1963.

[31] Kobeleva X, Firbank M, Peraza L, Gallagher P, Thomas A, Burn DJ, O'Brien J, Taylor JP (2017) Divergent functional connectivity during attentional processing in Lewy body dementia and Alzheimer's disease. Cortex 92, 8-18.

[32] Brier MR, Thomas JB, Snyder AZ, Benzinger TL, Zhang D, Raichle ME, Holtzman DM, Morris JC, Ances BM (2012) Loss of intranetwork and internetwork resting state functional connections with Alzheimer's disease progression. $J$ Neurosci 32, 8890-8899.

[33] Ding J, Chen K, Chen Y, Fang Y, Yang Q, Lv Y, Lin N, Bi Y, Guo Q, Han Z (2016) The left fusiform gyrus is a critical region contributing to the core behavioral profile of semantic dementia. Front Hum Neurosci 10, 215.

[34] Farb NAS, Grady CL, Strother S, Tang-Wai DF, Masellis M, Black S, Freedman M, Pollock BG, Campbell KL, Hasher L, Chow TW (2013) Abnormal network connectivity in frontotemporal dementia: Evidence for prefrontal isolation. Cortex 49, 1856-1873.

[35] Agosta F, Galantucci S, Valsasina P, Canu E, Meani A, Marcone A, Magnani G, Falini A, Comi G, Filippi M (2014) Disrupted brain connectome in semantic variant of primary progressive aphasia. Neurobiol Aging 35, 2646-2655.

[36] La Joie R, Landeau B, Perrotin A, Bejanin A, Egret S, Pélerin A, Mézenge F, Belliard S, de La Sayette V, Eustache F, Desgranges B, Chételat G (2014) Intrinsic connectivity identifies the hippocampus as a main crossroad between Alzheimer's and semantic dementia-targeted networks. Neuron 81, 1417-1428.

[37] Margulies DS, Kelly AMC, Uddin LQ, Biswal BB, Castellanos FX, Milham MP (2007) Mapping the functional connectivity of anterior cingulate cortex. Neuroimage 37, 579-588.

[38] Craddock RC, James GA, Holtzheimer PE 3rd, Hu XP, Mayberg HS (2012) A whole brain fMRI atlas generated via spatially constrained spectral clustering. Hum Brain Mapp 33, 1914-1928.

[39] Henke K (2010) A model for memory systems based on processing modes rather than consciousness. Nat Rev Neurosci 11, 523-532.

[40] Duss SB, Reber TP, Hänggi J, Schwab S, Wiest R, Müri RM, Brugger P, Gutbrod K, Henke K (2014) Unconscious relational encoding depends on hippocampus. Brain 137, 3355-3370.

[41] Orban P, Dansereau C, Desbois L, Mongeau-Pérusse V, Giguère C-É, Nguyen H, Mendrek A, Stip E, Bellec P (2018) Multisite generalizability of schizophrenia diagnosis classification based on functional brain connectivity. Schizophr Res 192, 167-171.

[42] Neary D, Snowden JS, Gustafson L, Passant U, Stuss D, Black S, Freedman M, KerteszA, Robert PH, Albert M, Boone K, Miller BL, Cummings J, Benson DF (1998) Frontotemporal lobar degeneration: A consensus on clinical diagnostic criteria. Neurology 51, 1546-1554.

[43] Gorno-Tempini ML, Hillis AE, Weintraub S, Kertesz A, Mendez M, Cappa SF, Ogar JM, Rohrer JD, Black S, Boeve BF, Manes F, Dronkers NF, Vandenberghe R, Rascovsky K, Patterson K, Miller BL, Knopman DS, Hodges JR, Mesulam MM, Grossman M (2011) Classification of primary progressive aphasia and its variants. Neurology 76, 1006-1014.

[44] World Health Organization (2004) International Statistical Classification of Diseases and Related Health Problems, World Health Organization.

[45] Grieder M, Crinelli RM, Jann K, Federspiel A, Wirth M, Koenig T, Stein M, Wahlund LO, Dierks T (2013) Correlation between topographic N400 anomalies and reduced cerebral blood flow in the anterior temporal lobes of patients with dementia. J Alzheimers Dis 36, 711-731.

[46] Andreotti J, Dierks T, Wahlund LO, Grieder M (2017) Diverging progression of network disruption and atrophy in Alzheimer's disease and semantic dementia. J Alzheimers Dis 55, 981-993.

[47] Chen Y, Chen K, Ding J, Zhang Y, Yang Q, Lv Y, Guo Q, Han Z (2017) Brain network for the core deficits of semantic dementia: A neural network connectivity-behavior mapping study. Front Hum Neurosci 11, 267.

[48] Afyouni S, Nichols TE (2018) Insight and inference for DVARS. Neuroimage 172, 291-312.

[49] Power JD, Barnes KA, Snyder AZ, Schlaggar BL, Petersen SE (2012) Spurious but systematic correlations in functional connectivity MRI networks arise from subject motion. Neuroimage 59, 2142-2154.

[50] Hurley RS, Bonakdarpour B, Wang X, Mesulam M-M (2015) Asymmetric connectivity between the anterior temporal lobe and the language network. J Cogn Neurosci 27, 464-473.

[51] Maldjian JA, Laurienti PJ, Kraft RA, Burdette JH (2003) An automated method for neuroanatomic and cytoarchitectonic atlas-based interrogation of fMRI data sets. Neuroimage $\mathbf{1 9}$, 1233-1239.

[52] Power JD, Mitra A, Laumann TO, Snyder AZ, Schlaggar BL, Petersen SE (2014) Methods to detect, characterize, and 
remove motion artifact in resting state fMRI. Neuroimage 84, 320-341.

[53] Chang C, Glover GH (2009) Effects of model-based physiological noise correction on default mode network anti-correlations and correlations. Neuroimage 47, $1448-1459$.

[54] Murphy K, Birn RM, Bandettini PA (2013) Resting-state fMRI confounds and cleanup. Neuroimage 80, 349-359.

[55] Miller GA, Chapman JP (2001) Misunderstanding analysis of covariance. J Abnorm Psychol 110, 40-48.

[56] Ashburner J, Friston KJ (2000) Voxel-based morphometry - the methods. Neuroimage 11, 805-821.

[57] Gaser C, Dahnke R (2016) CAT-a computational anatomy toolbox for the analysis of structural MRI data. Hum Brain Mapp 2016, 336-348.

[58] Friston KJ, Ashburner JT, Kiebel SJ, Penny WD, Nichols TE (2007) Statistical Parametric Mapping: The Analysis of Functional Brain Images, Elsevier Science.

[59] McDonough IM, Nashiro K (2014) Network complexity as a measure of information processing across resting-state networks: Evidence from the Human Connectome Project. Front Hum Neurosci 8, 409.

[60] Sormaz M, Jefferies E, Bernhardt BC, Karapanagiotidis T, Mollo G, Bernasconi N, Bernasconi A, Hartley T, Smallwood J (2017) Knowing what from where: Hippocampal connectivity with temporoparietal cortex at rest is linked to individual differences in semantic and topographic memory. Neuroimage 152, 400-410.

[61] Shtyrov Y, Pulvermüller F (2007) Early MEG activation dynamics in the left temporal and inferior frontal cortex reflect semantic context integration. J Cogn Neurosci 19, 1633-1642.

[62] Jobard G, Crivello F, Tzourio-Mazoyer N (2003) Evaluation of the dual route theory of reading: A metanalysis of 35 neuroimaging studies. Neuroimage 20, 693-712.

[63] Wirth M, Horn H, Koenig T, Razafimandimby A, Stein M, Mueller T, Federspiel A, Meier B, Dierks T, Strik W (2008) The early context effect reflects activity in the temporo-prefrontal semantic system: Evidence from electrical neuroimaging of abstract and concrete word reading. Neuroimage 42, 423-436.

[64] Mesulam M-M, Thompson CK, Weintraub S, Rogalski EJ (2015) The Wernicke conundrum and the anatomy of language comprehension in primary progressive aphasia. Brain 138, 2423-2437.

[65] Nilakantan AS, Voss JL, Weintraub S, Mesulam M-M, Rogalski EJ (2017) Selective verbal recognition memory impairments are associated with atrophy of the language network in non-semantic variants of primary progressive aphasia. Neuropsychologia 100, 10-17.

[66] Brunyé TT, Moran JM, Holmes A, Mahoney CR, Taylor HA (2017) Non-invasive brain stimulation targeting the right fusiform gyrus selectively increases working memory for faces. Brain Cogn 113, 32-39.

[67] Rangarajan V, Hermes D, Foster BL, Weiner KS, Jacques C, Grill-Spector K, Parvizi J (2014) Electrical stimulation of the left and right human fusiform gyrus causes different effects in conscious face perception. J Neurosci 34, 12828-12836.

[68] Mion M, Patterson K, Acosta-Cabronero J, Pengas G, Izquierdo-Garcia D, Hong YT, Fryer TD, Williams GB, Hodges JR, Nestor PJ (2010) What the left and right anterior fusiform gyri tell us about semantic memory. Brain 133, 3256-3268.
[69] Owen AM, Milner B, Petrides M, Evans AC (1996) A specific role for the right parahippocampal gyrus in the retrieval of object-location: A positron emission tomography study. J Cogn Neurosci 8, 588-602.

[70] Luck D, Danion J-M, Marrer C, Pham B-T, Gounot D, Foucher J (2010) The right parahippocampal gyrus contributes to the formation and maintenance of bound information in working memory. Brain Cogn 72, 255-263.

[71] Tondelli M, Wilcock GK, Nichelli P, De Jager CA, Jenkinson M, Zamboni G (2012) Structural MRI changes detectable up to ten years before clinical Alzheimer's disease. Neurobiol Aging 33, 825.e25-36.

[72] Bruen PD, McGeown WJ, Shanks MF, Venneri A (2008) Neuroanatomical correlates of neuropsychiatric symptoms in Alzheimer's disease. Brain 131, 2455-2463.

[73] Fernández-Matarrubia M, Matías-Guiu JA, Cabrera-Martín MN, Moreno-Ramos T, Valles-Salgado M, Carreras JL, Matías-Guiu J (2018) Different apathy clinical profile and neural correlates in behavioral variant frontotemporal dementia and Alzheimer's disease. Int J Geriatr Psychiatry 33, 141-150.

[74] Kumfor F, Landin-Romero R, Devenney E, Hutchings R, Grasso R, Hodges JR, Piguet O (2016) On the right side? A longitudinal study of left- versus right-lateralized semantic dementia. Brain 139, 986-998.

[75] Olson IR, McCoy D, Klobusicky E, Ross LA (2013) Social cognition and the anterior temporal lobes: A review and theoretical framework. Soc Cogn Affect Neurosci 8, 123-133.

[76] Dermody N, Wong S, Ahmed R, Piguet O, Hodges JR, Irish M (2016) Uncovering the neural bases of cognitive and affective empathy deficits in Alzheimer's disease and the behavioral-variant of frontotemporal dementia. $J$ Alzheimers Dis 53, 801-816.

[77] Birn RM (2012) The role of physiological noise in restingstate functional connectivity. Neuroimage 62, 864-870.

[78] Murphy K, Fox MD (2017) Towards a consensus regarding global signal regression for resting state functional connectivity MRI. Neuroimage 154, 169-173.

[79] Yu M, Linn KA, Cook PA, Phillips ML, McInnis M, Fava M, Trivedi MH, Weissman MM, Shinohara RT, Sheline YI (2018) Statistical harmonization corrects site effects in functional connectivity measurements from multi-site fMRI data. Hum Brain Mapp 39, 4213-4227.

[80] Yamashita A, Yahata N, Itahashi T, Lisi G, Yamada T, Ichikawa N, Takamura M, Yoshihara Y, Kunimatsu A, Okada N, Yamagata H, Matsuo K, Hashimoto R, Okada G, Sakai Y, Morimoto J, Narumoto J, Shimada Y, Kasai K, Kato N, Takahashi H, Okamoto Y, Tanaka SC, Kawato M, Yamashita O, Imamizu H (2019) Harmonization of restingstate functional MRI data across multiple imaging sites via the separation of site differences into sampling bias and measurement bias. PLoS Biol 17, e3000042.

[81] Welch LW, Doineau D, Johnson S, King D (1996) Educational and gender normative data for the Boston naming test in a group of older adults. Brain Lang 53, 260-266.

[82] Tombaugh TN, Kozak J, Rees L (1999) Normative data stratified by age and education for two measures of verbal fluency: FAS and animal naming. Arch Clin Neuropsychol 14, 167-177.

[83] Woods SP, Scott JC, Sires DA, Grant I, Heaton RK, Tröster AI, HIV Neurobehavioral Research Center Group (2005) Action (verb) fluency: Test-retest reliability, normative standards, and construct validity. J Int Neuropsychol Soc 11, $408-415$. 